\title{
KILLCANCER: \\ nanobody-targeted photodynamic therapy to kill cancer
}

Dr Sabrina Oliveira

Utrecht University, The Netherlands

Current cancer therapies often fail to cure patients. Ideally, a cancer therapy should locally eradicate the cancer and activate the immune system to create a memory and protect from recurrences.

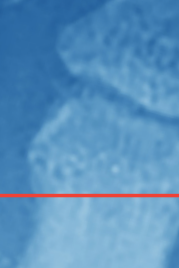

In photodynamic therapy (PDT), cance cells are killed with compounds name photosensitisers that are activated photosensitiser or light alone are harmless but can create toxicity and damage cells containing the photosensitiser when
combined.

Current protocols in the clinic start with the injection of a solution of photosensitiser into the bloodstream, allowing its distribution throughout the body. Two to four days later, the photosensitiser has been elliminated from normal cells but is retained in cancer cerls. At that point, the tumour is exposed to laser light of a specific wavelength. This activates the photosensitiser, that do toxic reactive oxysen species PDT has been described to activate the pationts from curnent PDT is only partlycancer specife, and petients remain sensitive to liect for several weeks after treatment.

Over the years, efforts have been made to improve the cancer specificity of PDT, for instance, by using antibodies to target the photosensitisers to cancer cells. This approach is currently being tested in clinical trials and has already been approved in Japan to treat head and neck cancer patients. In this case, cetuximab is the antibody used, which targets the epidermal growth factor receptor (EGFR), usually overexpressed on cancer cells in the head and neck region. This antibody is conjugated to the photosensitiser IRDye700DX, a phthalocyanine derivative that is watersoluble. Although this approach has been a significant improvement, further advancements are still possible.

Preclinical studies have shown that the antibody-photosensitiser conjugates are relatively large to penetrate and distribute homogeneously through tumours, preventing them from completely eradicating the cancer. Antibodies also circulate in the bloodstream for severa days, delaying light application and making photosensitivity a remaining issue. Dr Oliveira has been developing (since 2012) a new form of targeted PDT, using

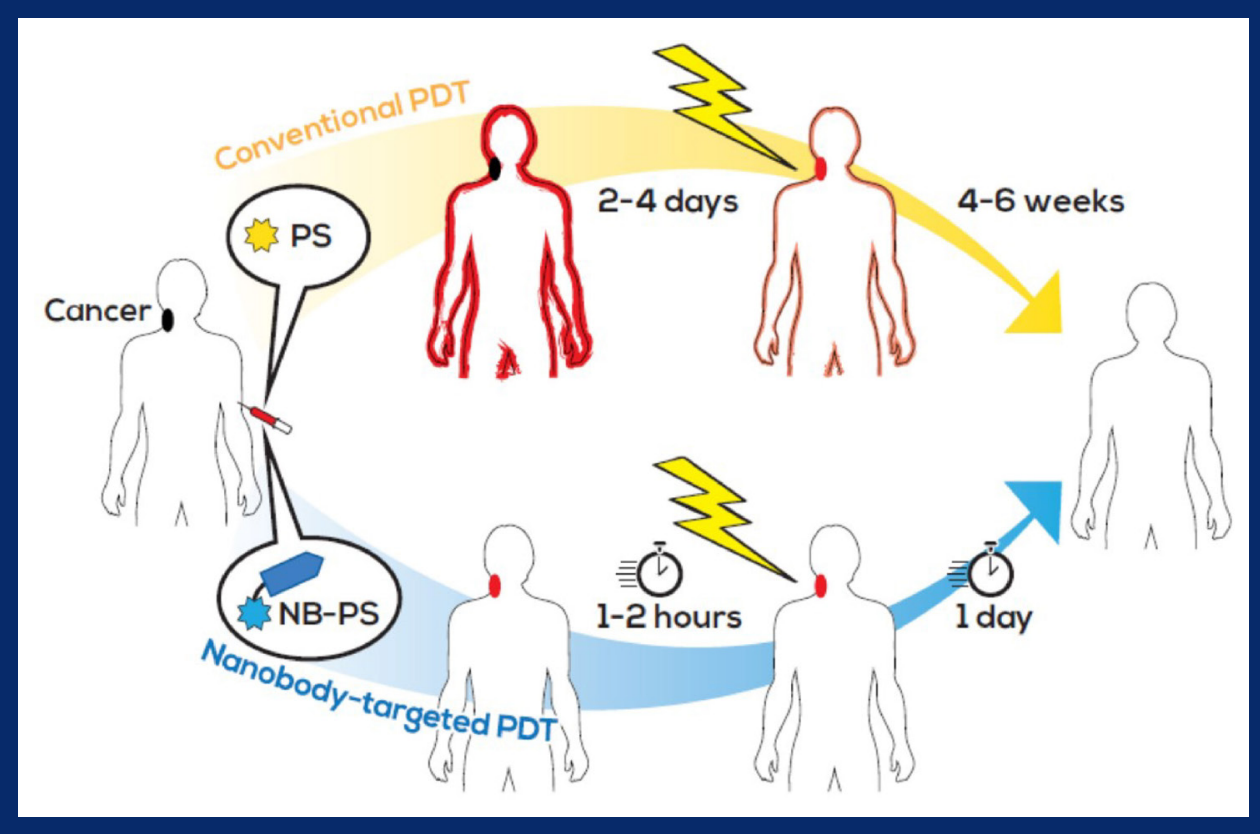

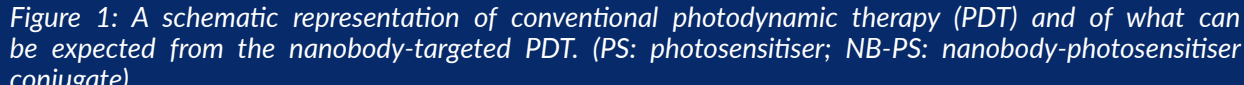

nanobodies to target the photosensitiser to cancer cells to solve all these issues. Nanobodies are small antibody fragments derived from a particular class of antibodies that exist in camelids. than conventional antibodies, and because of this small size: (i) nanobodies accumulate in tumours within 1-2 hours after intravenous administration; (ii) they distribute very well through a tumour mass; and (iii) they are rapidly eliminated, if not associated or bound to cells (Figure 1). Thus, nanobody-targeted PDT enables the application of light shortly after administration of the nanobodyphotosensitiser conjugate. Unlike the most traditional photosensitisers, the one used in this approach is watersoluble, i.e. the IRDye700DX as used in the antibody-photosensitiser conjugate tested in the clinic. Because of this, it does not stick randomly to every cell it the nanobody to

One of the seat advatases of PDT

One of the great advantages of PDT is
that the toxicity is only created where

light is applied; thus, it is not harmful to the patient's healthy tissues. By rendering the accumulation of the photosensitiser even more cancer specific, using are even lower. This makes targeted PDT an excellent alternative for patients with

umours in places that are too risky to perate on, for example, in the head and neck regions, because of the collateral damage that could occur. The second main advantage of PDT is that it can activate the patient's immune system, possibly inducing long-term protection against the recurrence of the cancer.

After our first in vitro studies (Heukers, 2014) in which nanobody-photosensitiser conjugates were shown to bind rapidly and specifically to cancer cells via EGFR, specifically leading to their destruction (Figure 2a), more recent studies have confirmed this approach can be expanded to other receptors overexpressed on cancer cells, namely the viral GPCR U28 in the context of glioblastomas (De Groof \& Mashayekhi, 2019), MET which is overexpressed in several malignancies (Heukers, 2019), and HER2 that was explored in the context of breast cancer Deken \& Kijanka, 2020).

The first studies conducted with experimental animals bearing human tumours showed that the nanobodyphotosensitiser conjugates, which are fluorescent, bind rapidly and specifically to the cancer cells and distribute homogeneously throughout the
tumour. One hour post-injection of the nanobody-photosensitiser conjugate, these tumours were illuminated, leading 

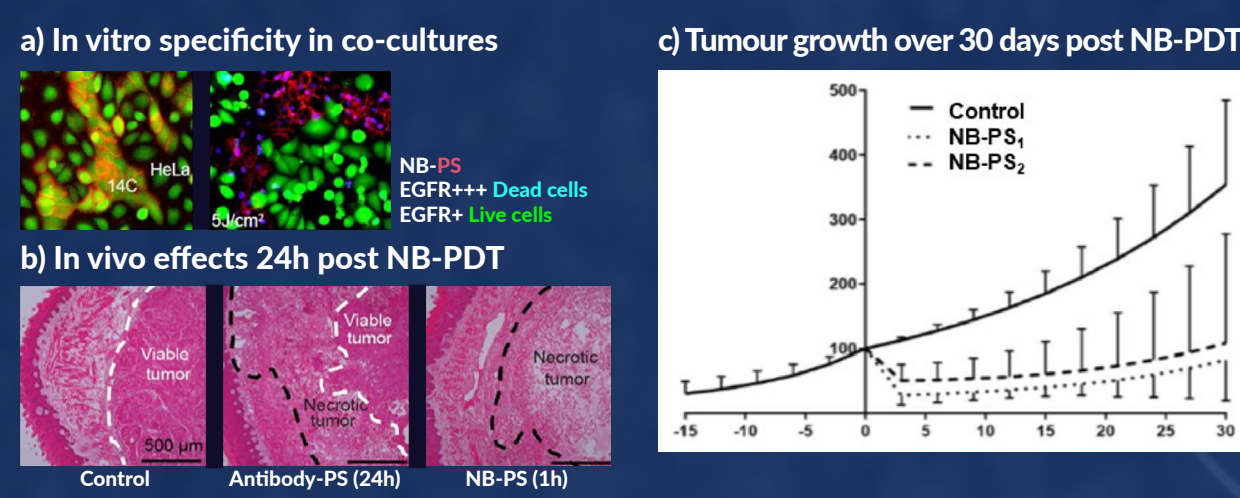

Figure 2: a) Nanobody-targeted PDD is selective to cells with overexpression of the tumour marker (e.g. EGFR), whereas normal cells remain alive. b) Proof of principle in vivo study showing extensive tumour damage
when nanobody-targeted PDT is applied, while arreas of viable tumeour are visible after antibody-targeted PDT (lllumination time post injection is indicated). c) Tumour volume over 30 days post nanobody-targeted
PDT, illustrating clear antitimour effects. (NB-PS: nanobody-photosensitiser conjugate; NB-PDT: nanobody
targeted photodynamic therapy).

Vascular effects trigsered by NB-PDT

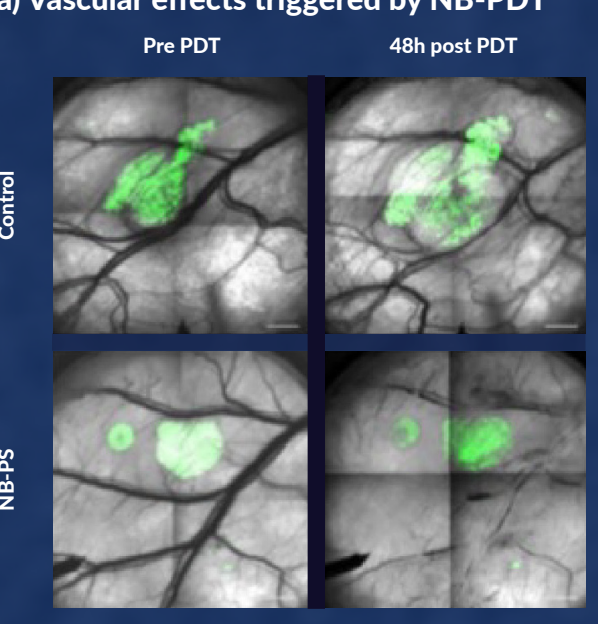

to approximately 90 per cent tumour damage (Figure 2b) (van Driel, 2016). A subsequent study investigated the effects on tumour growth over 30 days post-treatment (Deken \& Kijanka,
2020). In this study, the nanobody2020). In this study, the nanobodyphotosensitiser targeted HER2, and a clear shrinkage of the tumour was (Figure 2c). Many of these tumours did not regrow in the following weeks. Such a strong antitumour effect was only observed for the tumour model with the highest HER2 expression, highlighting how relevant the expression of the target is for the efficacy of the targeted therapy.

In order to investigate nanobody-targeted PDT in a setting closer to the clinic, cance and normal tissue organoids obtained from patients with head and neck cancer were employed (Driehuis \& Spelie, 2019). These organoids recapitulated the expression level of the tumour target, EGFR in this study, from the patient'
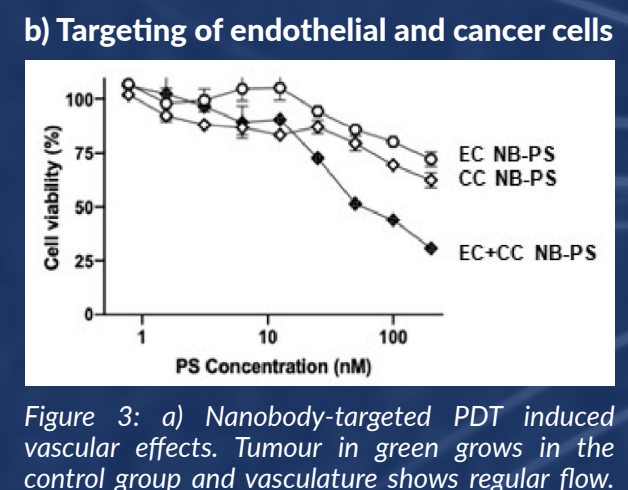
Treated tumour reduces in size and vasculature
presents disrupted flow. b) Targeting of endothelial presents disrupted flow. b) Targeting of endothell
and cancer cells potentiates the effect of nanoboci

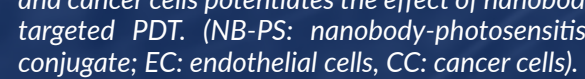

fresh tissue obtained. Therefore it was concluded that these organoids are useful model to investigate the efficacy tested, nanobody-targeted PDT induced cytotoxicity solely on cancer organoic while normal organoids remained alive, and it was superior to antibody-targeted PDT, further supporting the development of our approach.

In parallel studies, more insights wer obtained into the mechanism of tumo destruction triggered by nanobod targeted PDT. Next to the mosty due to membrane cell damage, vascular effects were also investigated. In dedicated study employing intravascul microscopy, we observed that while the vasculature was well visible and tumours were well perfused before treatment, (1) some cases with damage and leakage observed direct cell death by necrosis the vessels (Figure 3a) (de Bruijn, 2020). While these effects seemed permanent in the tumour vasculature, they appeared transient outside the tumour are

As the vascular effects could also aid in tumour destruction, we hypothesised that in areas where the tumour target was less available, it would be beneficial to also target the tumour vasculature for more effective destruction. For this, we have developed nanobody photosensitiser conjugates targeting Ditothelial cells. In vitro studies have confirmed that a more potent effect is obtained by the dual targeting of cancer and endothelial cells (Figure 3b) Mashayekhi, 2020). This combination remains to be studied in vivo.

Next to direct cell death and vascular effects, an immune response is described to contribute to cancer destruction in PDT. Also, in this targeted approach using nanobodies, this seems to apply.
Thus far, in vitro and ex vivo studies Thus far, in vitro and ex vivo studies
have confirmed that immunogenic cell have confirmed that immunogenic cell death occurs when cancer cells are
treated with nanobody-targeted PDT. treated with nanobody-targeted PDT.
This can lead to the maturation of This can lead to the maturation of dendritic cells, which subsequently are able to induce the proliferation of T cells (Beltrán Hernández, 2020). More studies
will follow to investigate the immune responses in more detail, namely with mmunocompetent mice.

Finally, the team aimed to bring chonoty -targeted PDT to the veterinary clinic by applying this treatment to cats with oral cancer. For this, patient materia available at the pathology department of the faculty of veterinary sciences was tested for the presence of EGFR (Beltrán Hernández, 2021). It was confirmed that EGFR could be used as a target for the nanobodies to deliver the photosensitiser in cats with oral cancer, as nine of the ten cases analysed expressed moderate to high EGFR in the tumour (Figure 4a). Similar levels were also observed at the epithelium, though veterinarians reassured this would not be a problem as light is locally applied, and the epithelium should rapidly regenerate if damaged.

A nanobody-photosensitiser was identified to be effective in killing feline cancer cells a) EGFR is overexpressed in feline oral cancer

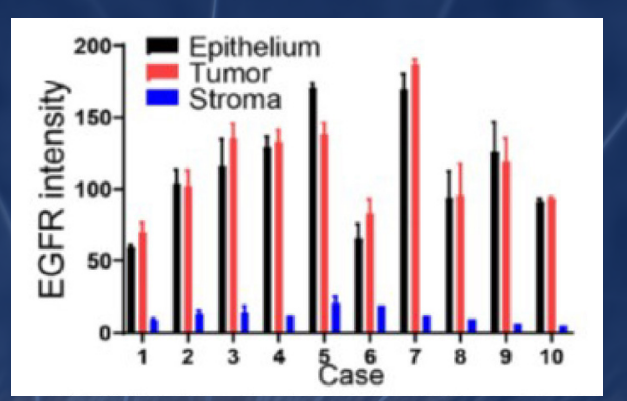

Figure 4: a) EGFR confirmed as a target for
nanobody-targeted PDT in feline oral cancer. The presence of
epithelium of ten cases of oral cancer from previous cats, patients at the veterinary clinic, b) Nanobody
targeted PDT is effective solely on SCCF felif

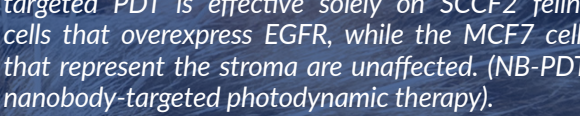

(Figure 4b). This approach will be tested in cats with oral cancer throughout 2022.

KILLCANCER has scientifically advanced the field of targeted PDT by providing

b) NB-PDT is effective on feline

SCCF2 cells
MCF7 cells

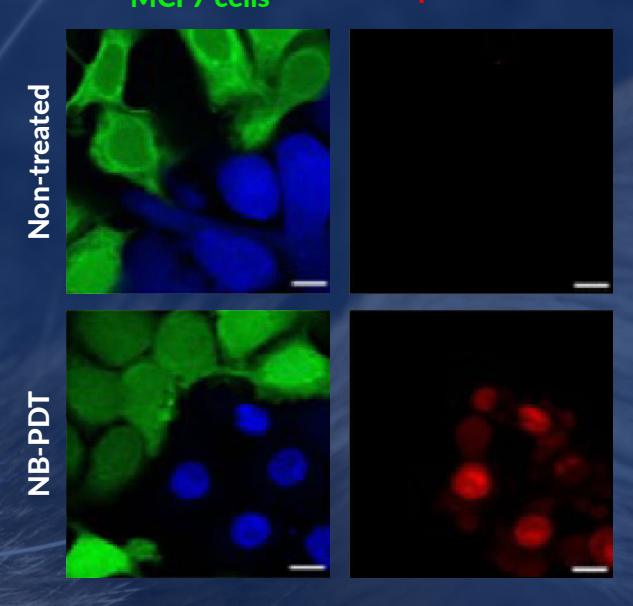

action and the feasibility of this approach to treat cancer. Ongoing studies focus on its translation to the clinic, starting with the veterinary clinic, aiming to ultimate mprove current cancer treatment.

\section{References}

Beltrán Hernández, I., Angelier, M., Del Buono D'Ondes, T., Di Maggio, A, Yu, Y. and Oliveira, S. (2020)
The Potential of Nanobody Targeted Photodynamic Therapy to Trigger Immune Responses, Cancers,
12(4), 978 . doi: $10.3390 /$ cancers12040978.

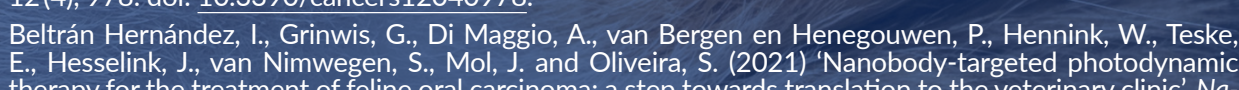

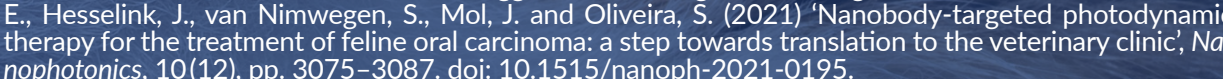

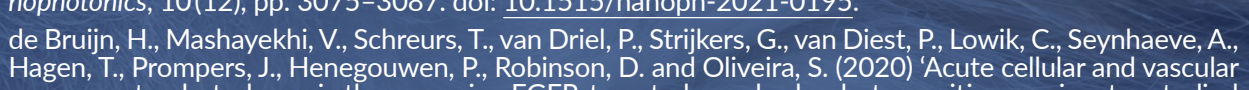

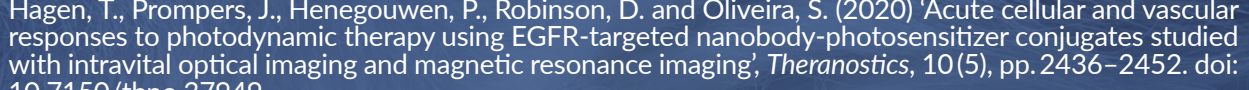

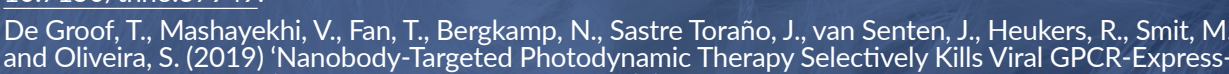
ing Glioblastoma Cells,' Molecular Pharmaceutics, 16(7), pp. 3145-3156. doi: 10.1021/acs.molpharma-
ceut Deken, M., Kijanka, M., Beltrán Hernández, I., Slooter, M., de Brujin, H., van Diest, P., van Bergen en
Henegouwen, P., Lowik, C., Robinson, D., Vahrmeijer, A. and Oliveira, S. (2020) Nanobody-targeted photodynamic therapy induces significant tumor regression of trastuzumab-resistant HER2-positive breast
cancer, after a single treatment session', Journal of Controlled Release, 323, pp. 269-281. do: $10.1016 / \mathrm{j}$. Driehuis, E, Spelier, S., Beltrán Hernández, I., de Bree, R., M. Willems, S., Clevers, H. and Oliveira, S. (2019)

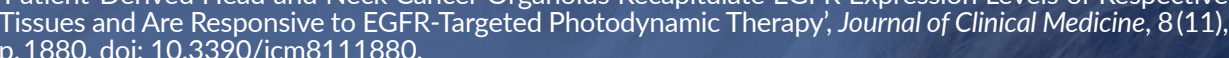

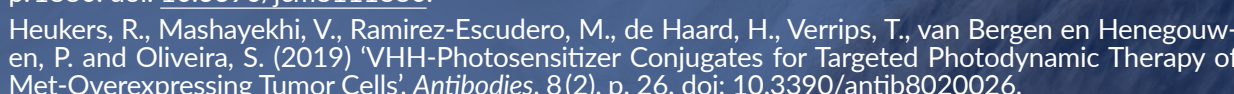
Met-Overexpressing Tumor Cells, Antibodies, 8(2), p. 26. doi: 10.3390/antib8020026. Heukers, R, van Bergen en Henegouwen, P. and Oliveira, S. (2014) 'Nanobody-photosensitizer conju-
gates for targeted photodynamic therapy', Nanomedicine: Nanotechnology, Biology and Medicine, 10(7).
pp. 1441-1451. doi: $10.1016 /$ /.nano.2013.12.007.

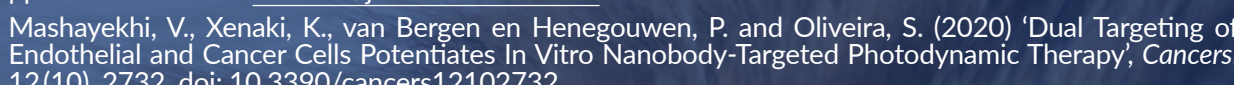
Mashayekhi, V., Xenaki, K. T., van Bergen en Henegouwen, P. M. P. and Oliveira, S. (2020) 'Dual targeting
of endothelial and cancer cells potentitites in vitro nanobody-targeted photodynamic therapy', Cancers,
12 (1)

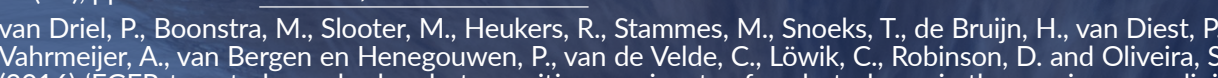

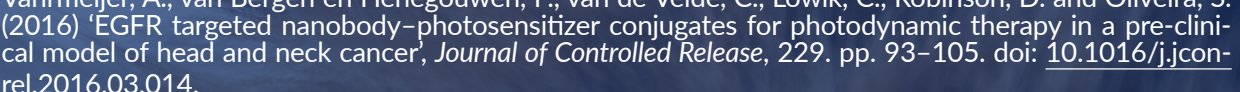

\section{PROJECT NAME}

KILLCANCER: nanobody-targeted

photodynamic therapy to kill cancer

PROJECT SUMMARY

KILLCANCER aims to better understand and advance a new form of targeted photodynamic therapy, a cancer therapy that through This new form makes use of nanobodies to render the therapy more effective and cancer
specific. We have obtained encouraging specific. We have obtained encouraging
results that support further testing in the veterinary clinic.
vesults

\section{PROJECT PARTNERS}

Important partners in this project are the Utrecht University, the Utrecht and Leiden University medical centres, and the Erasmus Medical Center in Rotterdam. These have ensured the clinical relevance of our studies and contributed to a faster translation to the veterinary clinic.

\section{PROJECT LEAD PROFILE}

Dr Sabrina Oliveira is, since 2019, an Associate Professor at Utrecht University, with a shared position between the of Pharmaceutical Sciences. Her research group named Molecular Targeted Therapies started in 2016, funded by the ERC Starting Grant KILLCANCER. Her group continues to focus on improving therapies by making these more target-specific using nanobodies.

\section{PROJECT CONTACTS}

Dr Sabrina Oliveira

Padualaan 8, 3584CH Utrecht,

The Netherlands

푱 +316034103460

\.oliveira@uu.ni

https://cellbiology.science.uu.nl/ molecular-targeted-therapies/

• @Sabrina_MTT

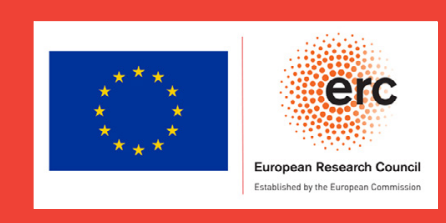

FUNDING

European Research Council (ERC) under the
ERro European Union's Horizon 2020 research and innovatic nor
677582. 